\title{
A Nomogram Based on Clinicopathologic Features and Preoperative Hematology Parameters to Predict Occult Peritoneal Metastasis of Gastric Cancer: A Single-Center Retrospective Study
}

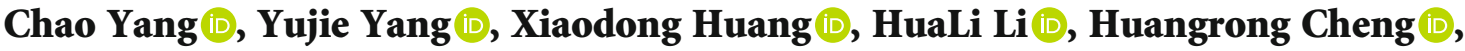 \\ Shilun Tong $\mathbb{D}$, and Yongbin Zheng $\mathbb{D}$ \\ Department of Gastrointestinal Surgery, Renmin Hospital of Wuhan University, Wuhan, 430060 Hu Bei Province, China \\ Correspondence should be addressed to Yongbin Zheng; yongbinzheng@whu.edu.cn
}

Received 16 September 2019; Revised 22 October 2020; Accepted 22 November 2020; Published 10 December 2020

Academic Editor: Marcello Ciaccio

Copyright (c) 2020 Chao Yang et al. This is an open access article distributed under the Creative Commons Attribution License, which permits unrestricted use, distribution, and reproduction in any medium, provided the original work is properly cited.

\begin{abstract}
Background. In patients with gastric cancer (GC), peritoneal metastasis is an indication of the end stage and often indicates a poor outcome. The diagnosis of peritoneal metastasis, especially occult peritoneal metastasis (OPM), remains a challenge for surgeons. This study was designed to explore the relationship between OPM and clinicopathological characteristics and preoperative hematological parameters in patients with GC and to develop a nomogram to predict the probability of OPM before surgery. Methods. A total of 672 patients with GC from our center were included, including 583 OPM-negative and 89 OPM-positive patients. These patients were divided into training and validation groups based on when they received treatment. OPM was diagnosed during surgery in patients without any signs of metastasis through imaging examination. Predictive factors were screened by least absolute shrinkage and selection operator logistic regression of all 18 characteristics. The nomogram of OPM was constructed based on these filtered variables. The discriminative and calibration performance of the model were simultaneously evaluated. Results. A total of six variables, including tumor size, degree of differentiation, depth of invasion, Glasgow prognosis score, and plasma levels of CA125 and fibrinogen, were selected for integration into the final predictive nomogram. The area under curve (AUC) of the nomogram with six factors was 0.906 (95\% confidence interval (CI): 0.872 0.941 ) and 0.889 (95\% CI: 0.795-0.984) in the training and validation groups, respectively. Calibration plots of the nomogram in the two sets revealed a good consistency between predicted and actual probabilities. Decision curve analysis showed that the nomogram had a positive net benefit among all threshold probabilities between $0 \%$ and $82 \%$. This nomogram was superior to models incorporating only clinicopathologic or hematologic features. Conclusion. Both clinicopathological and preoperative hematological parameters are significantly associated with OPM. The nomogram constructed with six factors could be used to calculate the probability of OPM and identify the high-risk population in GC. This may be helpful for early detection of OPM in patients with GC.
\end{abstract}

\section{Introduction}

Gastric carcinoma (GC) is the fifth most common cancer and one of the leading causes of cancer-related death worldwide, especially in East Asia [1]. Peritoneal metastasis, the major style of distant metastasis in patients with GC, is responsible for over half of the mortality from GC [2]. In GC, approximately $10 \%-30 \%$ of peritoneal metastasis can only be discovered during laparoscopy $[3,4]$. In patients with GC, occult peritoneal metastasis (OPM) is a special type of peritoneal metastasis that is diagnosed during surgery without any signs of metastasis through imaging examination [5]. OPM includes two conditions, namely, cytology positive (CY1) and macroscopic metastatic lesions (P1) [6]. Hence, OPM could be considered as an early stage of metastasis. Patients with OPM have a more aggressive treatment regimen and better outcome than do those with extensive metastatic lesions $[6,7]$. Consequently, accurate and timely preoperative evaluation is crucial for doctors to choose the most appropriate therapies. However, the early detection of 
metastatic sites, especially for potential peritoneal metastases, remains a challenge for surgeons [8]. Conventional imaging examinations for peritoneal metastasis detection, including computerized tomography (CT) or magnetic resonance imaging (MRI), have insufficient sensitivity and specificity [9-11]. Although diagnostic laparoscopy is a minimally invasive examination with many advantages, clinicians took conservative attitudes on its wide application for peritoneal metastasis detection because of its procedure-related complications [12-14]. Therefore, it is urgent to develop an appropriate noninvasive preoperative detection method as a complementary diagnostic tool for OPM in GC by combining multiple parameters.

Previous studies have indicated that the inflammatory level is closely related to tumor proliferation, invasion, and metastasis in GC $[15,16]$. The most commonly used indicators of host inflammation level, including neutrophil-tolymphocyte ratio (NLR), platelet-to-lymphocyte ratio (PLR), and Glasgow prognosis score (GPS), are considered novel predictors in many cancer types [17-19]. Using simple inflammatory cell counts for predicting cancer progression and for prognostic assessment has gradually received increased attention in GC $[20,21]$. Whether NLR, PLR, and GPS can predict OPM in GC has not been assessed. Moreover, correlations between coagulation factors and cancers can date back for over a century. More than 95\% of metastatic malignancies have coagulation function abnormalities, and cancer-induced hypercoagulability might accelerate tumor progression and dissemination [22]. Therefore, coagulation data should also be considered in this prediction model. A systematic review suggested that although serum tumor biomarkers cannot detect cancer in its early stage, they are useful for monitoring recurrence and predicting peritoneal/liver metastases [23]. Changes in the levels of tumor biomarkers usually occur 2-3 months before imaging abnormalities can be detected. Therefore, these new findings provide a theoretical basis for OPM detection as early as possible by combining multiple parameters.

In this study, we reviewed patients with GC and OPM in our center and explored factors associated with OPM. The aim of this study was to establish a noninvasive and lowcost prediction model that might be useful for the early detection of OPM.

\section{Methods}

2.1. Patients. This is a retrospective study. Patients who underwent laparoscopy from January 2014 to April 2019 in the Department of Gastrointestinal Surgery, Renmin Hospital of Wuhan University, were included. All patients were diagnosed with GC by upper gastrointestinal endoscopy and pathological examination.

The inclusion criteria are as follows: (1) gastric cancer diagnosed by pathology; (2) no evidence indicating tumor invasion of adjacent organs or distant metastasis by preoperative evaluation; and (3) patients were deemed to be suitable for potential curative resection by a multidisciplinary team. The exclusion criteria are as follows: (1) patients had other cancers at the same time; (2) patients received radiotherapy and/or chemotherapy before surgery; (3) patients had any other serious infectious diseases; (4) patients had any blood system diseases and/or had taken medicine that affected coagulation function; and (5) patients had undergone surgery in emergency condition. Included patients were divided into two sets based on the different treatment times.

Patients treated between January 2014 and July 2018 were the training group, and patients treated from August 2018 to April 2019 were the validation group. This study was approved by the Ethics Committee of Renmin Hospital of Wuhan University, and follows the principles of the Declaration of Helsinki. Patient information was anonymized and treated confidentially.

2.2. Variables. Patients were assessed before surgery by physical examinations, chest X-ray, abdominal and pelvic contrast-enhanced CT, and blood tests. Venous blood samples were obtained from the patients the morning after admission. Preoperative laboratory data was acquired, including blood cell count, C-reactive protein (CRP), albumin, gastrointestinal tumor biomarkers, and coagulation function tests. Gender and age were also recorded as general characteristics. Tumor-related data included primary tumor location, tumor size, and preoperative clinical staging as determined by CT. Histopathological parameters, including histological types and differentiation degree, were assessed using the preoperative biopsy tissues.

Gastric signet ring cell carcinoma or mucinous adenocarcinoma were defined as nonadenocarcinoma. Clinical T4 was defined as serosa invasion, and clinical T1, T2, and T3 were defined as nonserosa invasion. The GPS ranged from 0 to 2 . GPS was a 2 when the CRP level $\geq 10 \mathrm{mg} / \mathrm{L}$ and albumin level $<35 \mathrm{mg} / \mathrm{L}$. If only one of CRP or albumin was abnormal, the GPS was 1 . If neither CRP nor albumin was abnormal, the GPS was 0.

2.3. Surgical Procedure. The laparoscopy procedure was performed as previously described [12]. All laparoscopy procedures were performed by experienced surgeons at the Department of Gastrointestinal Surgery at Renmin Hospital, Wuhan University.

2.4. OPM Diagnostic Criteria. OPM was diagnosed when there was no evidence of peritoneal metastasis before surgery, but such metastasis was confirmed during surgery, including CY1 and P1. The weakly diagnostic sensitivity of frozen sections during surgery meant that CY1 and P1 diagnosis depended on exhaustive pathological examination.

2.5. Statistical Analysis. Statistical analysis was conducted using the IBM SPSS Statistics software (version 22) and R (version 3.6.0.). The Youden index was used as the optimal cutoff value of each continuous parameter assessed by the receiver operating characteristic (ROC) curve. The chisquare or Fisher tests were used to analyze categorical variables between OPM-positive and OPM-negative groups. Predictive factors were selected by least absolute shrinkage and selection operator (LASSO) regression, and the OPM nomogram prediction models were constructed based on these factors. The area under the curve (AUC) of ROC curves 
TABLE 1: Demographic and clinicopathological characteristics of patients.

\begin{tabular}{|c|c|c|c|c|c|c|}
\hline \multirow{2}{*}{ Various } & \multicolumn{3}{|c|}{ Training group } & \multicolumn{3}{|c|}{ Validation group } \\
\hline & $\mathrm{OPM}(+)$ & OPM (-) & $P$ value & $\mathrm{OPM}(+)$ & OPM (-) & $P$ value \\
\hline Gender, no. (\%) & & & 0.555 & & & 0.135 \\
\hline Female & $21(28.8)$ & $146(32.2)$ & & $9(56.3)$ & $48(36.9)$ & \\
\hline Male & $52(71.2)$ & $307(67.8)$ & & $7(43.8)$ & $82(63.1)$ & \\
\hline Age, no. (\%) & & & 0.900 & & & 0.512 \\
\hline$<60$ & $36(49.3)$ & $227(50.1)$ & & $7(43.8)$ & $61(46.9)$ & \\
\hline$\geq 60$ & $37(50.7)$ & $226(49.9)$ & & $9(56.3)$ & $69(53.1)$ & \\
\hline Location, no. (\%) & & & 0.209 & & & 0.430 \\
\hline Lower third & $36(49.3)$ & $259(57.2)$ & & $11(68.8)$ & $74(56.9)$ & \\
\hline Upper/middle third & $37(50.7)$ & $194(42.8)$ & & $5(31.3)$ & $56(43.1)$ & \\
\hline Size, no. (\%) & & & $<0.001$ & & & 0.003 \\
\hline$<5 \mathrm{~cm}$ & $20(27.4)$ & $311(68.7)$ & & $5(31.3)$ & $93(71.5)$ & \\
\hline$\geq 5 \mathrm{~cm}$ & $53(72.6)$ & $142(31.3)$ & & $11(68.8)$ & $37(28.5)$ & \\
\hline Pathological type, no. (\%) & & & 0.971 & & & 0.622 \\
\hline Adenocarcinoma & $62(84.9)$ & $384(84.8)$ & & $14(87.5)$ & $120(92.3)$ & \\
\hline Nonadenocarcinoma & $11(15.1)$ & $69(15.2)$ & & $2(12.5)$ & $10(7.7)$ & \\
\hline Differentiated degree, no. (\%) & & & $<0.001$ & & & 0.102 \\
\hline High/middle & $9(12.3)$ & $203(44.8)$ & & $5(31.3)$ & $67(51.5)$ & \\
\hline Poorly & $64(87.7)$ & $250(55.2)$ & & $11(68.8)$ & $63(48.5)$ & \\
\hline Depth of invasion, no. (\%) & & & $<0.001$ & & & $<0.001$ \\
\hline Nonserosal invasion & $13(17.8)$ & $305(67.3)$ & & $3(18.8)$ & $85(65.4)$ & \\
\hline Serosal invasion & $60(82.2)$ & $148(32.7)$ & & $13(81.3)$ & $45(34.6)$ & \\
\hline
\end{tabular}

OPM (+): with occult peritoneal metastasis. OPM (-): without occult peritoneal metastasis. Gastric signet ring cell carcinoma or mucinous adenocarcinoma was defined as nonadenocarcinoma. cT4 was defined as serosal invasion, and cT1, cT2, and cT3 were defined as nonserosal invasion.

was used to evaluate the prediction model discrimination capability, and the Hosmer-Lemeshow test was used to measure goodness of fit. The calibration capability of the model was evaluated by bootstrap resampling, and the decision curve was used to estimate the clinical net benefit for patients. ROCs were compared using the DeLong test. All $P$ values were two-sided and $P<0.05$ was considered statistically significant.

\section{Results}

3.1. Clinical Characteristics and Hematologic Parameters of Patients with GC and OPM. A total of 672 patients with GC who met the inclusion criteria were included in this study. This included 526 in the training group and 146 in the validation group. In the training group, 73 cases were OPM positive and 453 cases were OPM negative. In the validation group, 130 cases were OPM negative and 16 cases were OPM positive. The OPM-positive rate in the training and validation groups were $13.9 \%$ and $10.9 \%$, respectively, and did not significantly differ $(P=0.157)$.

Univariate analysis results revealed that clinicopathological characteristics, including tumor size and depth of invasion, significantly correlated with OPM $(P<0.05$; Table 1$)$. Moreover, OPM was significantly associated with many hematological parameters (Table 2).
3.2. Feature Selection. All 18 potential predictors were incorporated into the LASSO logistic regression model for the 526 patients in the training set (Figure 1). Then, six predictors with nonzero coefficients were selected when lambda $=-3.082$ (lambda $\cdot 1 \mathrm{se})$. The six features were tumor size, degree of differentiation, depth of invasion, GPS, and plasma levels of CA125 and fibrinogen.

3.3. Construction of the Nomogram for Predicting OPM in Patients with GC. Those six predictors were added into the final nomogram (named Model A, Figure 2). Each factor in the nomogram was assigned a weighted score. For each patient, the total points were associated with the probability of OPM. Considering that some included parameters mentioned above may not be assessed in other centers, two models with only clinicopathological or hematological features (Model B and Model C, respectively) were also built and evaluated simultaneously.

3.4. Nomogram Performance in the Training and Validation Groups. The coefficients of variables in three models are shown in Table 3. The combined model (Model A) had lower Akaike information criterion (AIC) than did Model B and Model C, indicating the best fit. The categorical net reclassification improvement (NRI) revealed that Model A had better predictive value than did Model B and Model C in the training cohort (Model A vs. Model B: 0.160 (95\% CI: 0.067- 
TABLE 2: Univariate analysis of preoperative hematology parameters in groups with or without occult peritoneal metastasis.

\begin{tabular}{|c|c|c|c|c|c|c|}
\hline \multirow{2}{*}{ Various } & \multicolumn{2}{|c|}{ Training group } & \multirow{2}{*}{$P$ value } & \multicolumn{2}{|c|}{ Validation group } & \multirow{2}{*}{$P$ value } \\
\hline & $\mathrm{OPM}(+)$ & OPM (-) & & OPM $(+)$ & OPM (-) & \\
\hline NLR & & & $<0.001$ & & & 0.797 \\
\hline$<2$ & $20(27.4)$ & $224(49.4)$ & & $9(11.7)$ & $68(52.3)$ & \\
\hline$\geq 2$ & $53(72.6)$ & $229(50.6)$ & & $7(10.1)$ & $62(47.7)$ & \\
\hline PLR & & & $<0.001$ & & & 0.585 \\
\hline$<165$ & $33(45.2)$ & $302(66.7)$ & & $9(56.3)$ & $84(64.6)$ & \\
\hline$\geq 165$ & $40(54.8)$ & $151(33.3)$ & & $7(13.2)$ & $46(35.4)$ & \\
\hline CEA & & & 0.049 & & & 0.530 \\
\hline$<2.5 \mathrm{ng} / \mathrm{mL}$ & $49(67.1)$ & $352(77.7)$ & & $11(68.8)$ & $101(77.7)$ & \\
\hline$\geq 2.5 \mathrm{ng} / \mathrm{mL}$ & $24(32.9)$ & $101(22.3)$ & & $5(31.3)$ & $29(22.3)$ & \\
\hline CA199 & & & 0.001 & & & 0.521 \\
\hline$<30 \mathrm{U} / \mathrm{mL}$ & $46(63.0)$ & $364(80.4)$ & & $14(87.5)$ & $109(83.8)$ & \\
\hline$\geq 30 \mathrm{U} / \mathrm{mL}$ & $27(37.0)$ & $89(19.6)$ & & $2(12.5)$ & $21(16.2)$ & \\
\hline CA125 & & & $<0.001$ & & & 0.003 \\
\hline$<14 \mathrm{U} / \mathrm{mL}$ & $16(21.9)$ & $329(72.6)$ & & $3(18.8)$ & $77(59.2)$ & \\
\hline$\geq 14 \mathrm{U} / \mathrm{mL}$ & $57(78.1)$ & $124(27.4)$ & & $13(81.3)$ & $53(40.8)$ & \\
\hline GPS & & & $<0.001$ & & & 0.001 \\
\hline 0 & $20(27.4)$ & $196(43.3)$ & & $3(18.8)$ & $44(33.8)$ & \\
\hline 1 & $34(46.6)$ & $230(50.8)$ & & $5(31.3)$ & $79(60.8)$ & \\
\hline 2 & $19(26.0)$ & $27(6.0)$ & & $8(53.3)$ & $7(5.4)$ & \\
\hline PT & & & 0.811 & & & 0.670 \\
\hline$<12 \mathrm{sec}$ & $54(74.0)$ & $341(75.3)$ & & $14(87.5)$ & $117(90.0)$ & \\
\hline$\geq 12 \mathrm{sec}$ & $19(26.9)$ & $112(24.7)$ & & $2(12.5)$ & $13(10.0)$ & \\
\hline APTT & & & 0.467 & & & 0.566 \\
\hline$<28 \mathrm{sec}$ & $44(60.3)$ & $293(64.7)$ & & $10(62.5)$ & $92(70.8)$ & \\
\hline$\geq 28 \mathrm{sec}$ & $29(39.7)$ & $160(35.3)$ & & $6(37.5)$ & $38(29.2)$ & \\
\hline $\mathrm{TT}$ & & & 0.219 & & & 0.786 \\
\hline$<18 \mathrm{sec}$ & 47 (64.4) & $257(56.7)$ & & $11(68.8)$ & $82(63.1)$ & \\
\hline$\geq 18 \mathrm{sec}$ & $26(35.6)$ & $196(43.3)$ & & $5(31.3)$ & $48(36.9)$ & \\
\hline FIB & & & $<0.001$ & & & 0.005 \\
\hline$<3 \mathrm{~g} / \mathrm{L}$ & $26(35.6)$ & $278(61.4)$ & & $4(25.0)$ & $83(63.8)$ & \\
\hline$\geq 3 \mathrm{~g} / \mathrm{L}$ & 47 (64.6) & 175 (38.6) & & $12(75.0)$ & $47(36.2)$ & \\
\hline D-dimer & & & 0.078 & & & 0.054 \\
\hline$<0.5 \mathrm{mg} / \mathrm{L}$ & $36(49.3)$ & $273(60.3)$ & & $6(37.5)$ & $84(64.6)$ & \\
\hline$\geq 0.5 \mathrm{mg} / \mathrm{L}$ & $37(50.7)$ & $180(39.7)$ & & $10(62.5)$ & $46(35.4)$ & \\
\hline
\end{tabular}

OPM (+): with occult peritoneal metastasis; OPM (-): without occult peritoneal metastasis; NLR: neutrophil-to-lymphocyte ratio; PLR: platelet-to-lymphocyte ration; CEA: carcinoembryonic antigen; CA199: carbohydrate antigen 19-9; CA125: carbohydrate antigen 125; GPS: Glasgow prognosis score; PT: prothrombin time; APTT: activated partial thromboplastin time; TT: thrombin time; FIB: fibrinogen.

0.253), $P<0.001$; Model A vs. Model C: 0.177 (95\% CI: $0.060-$ $0.293), P=0.003)$. In the validation group, the categorical NRI revealed that Model $\mathrm{A}$ had better predictive value than did Model C, but was not markedly better than Model B (Model A vs. Model C: 0.171 (95\% CI: 0.001-0.341), $P=0.049$; Model A vs. Model B: 0.093 (95\% CI: -0.128 to -0.315$), P=0.409$ ).

The predictive accuracy of the nomogram was visually displayed by ROC curves (Figure 3). In the training group, Model A yielded the highest AUC (0.906 (95\% CI: $0.872-$ 0.941), indicating that this model has good discrimination and reliable ability as a predictive tool for OPM. The AUCs for Models B and C were 0.828 (95\% CI: 0.781-0.875) and
0.832 (95\% CI: 0.778-0.886), respectively. The DeLong test revealed that the AUCs significantly differed between Model $\mathrm{A}$ and the other two models (both $P<0.001$ for Model A vs. Model B or Model C). In the validation group, Model A had the highest AUC (0.889 (95\% CI: 0.795-0.984), while Models $\mathrm{B}$ and C had AUCs of 0.827 (95\% CI: 0.730-0.925) and 0.845 (95\% CI: 0.748-0.943). The DeLong test revealed statistically significant differences in the AUCs of Model A and Model B $(P=0.046)$, but not between Model $A$ and Model $C$ $(P=0.209)$.

The calibration plot suggested a good concordance between the predicted and observed values for Model A in 


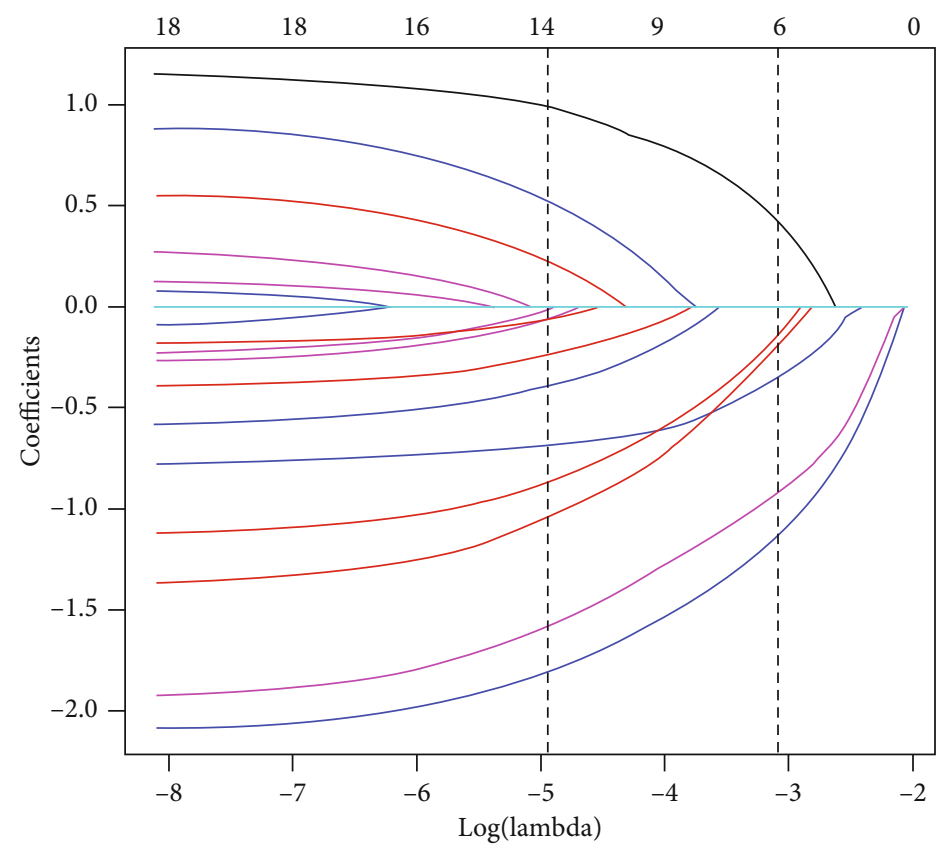

(a)

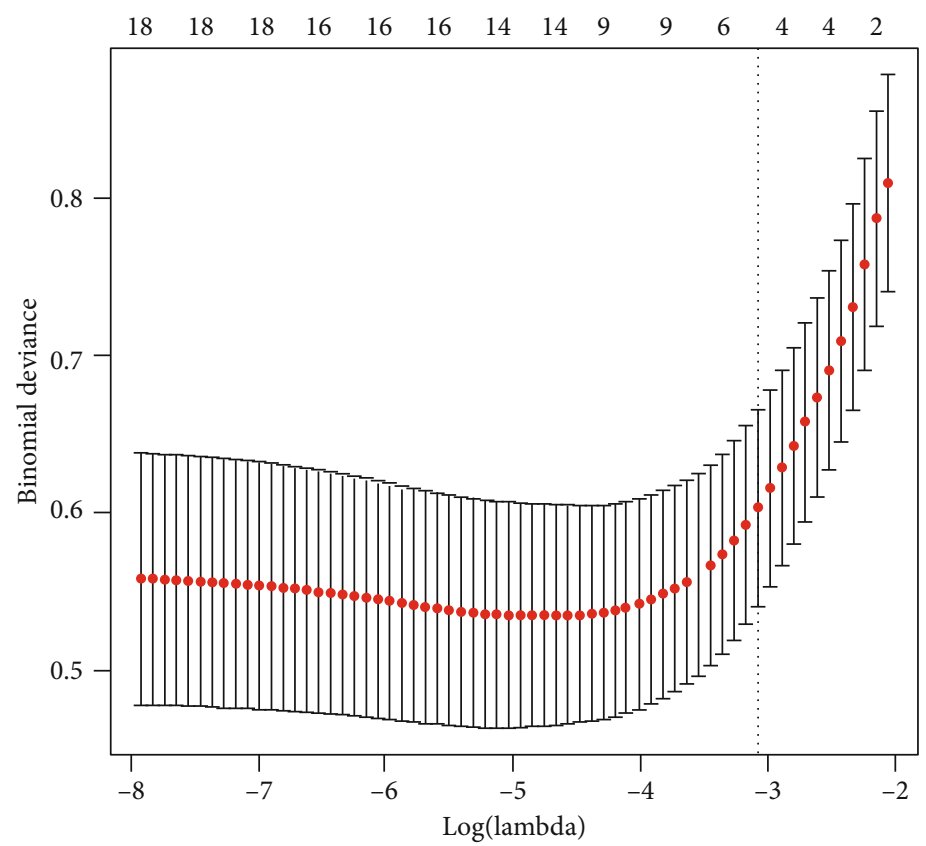

(b)

FIGURE 1: Predictive features were selected using the LASSO binary logistic regression model. (a) LASSO coefficient of the 18 OPM-associated predictors. (b) Feature selection using the LASSO model and 10-fold cross-validation via minimum criteria. The model had excellent performance and the least number of independent variables when lambda was -3.082.

both the training and validation sets (Figure 4 ). This was supported by the Hosmer-Lemeshow test results $\left(\chi^{2}=5.180\right.$, $P=0.819 ; \chi^{2}=3.359, P=0.948$ for training and validation sets, respectively).

3.5. Clinical Use. Decision curve analysis was based on the three nomogram models in the training set (Figure 5). Model A had the maximum clinical net benefit. The published liter- ature indicated that patients would need to receive specialized interventions (usually laparoscopy scheme) when the preoperative predicted probabilities of peritoneal metastasis are greater than 30\% [12]. Compared with patients who underwent all laparoscopic or nonlaparoscopic interventions, the patient could obtain an additional clinical net benefit of approximately $20 \%$ by the use of Model A for clinical decision-making. 


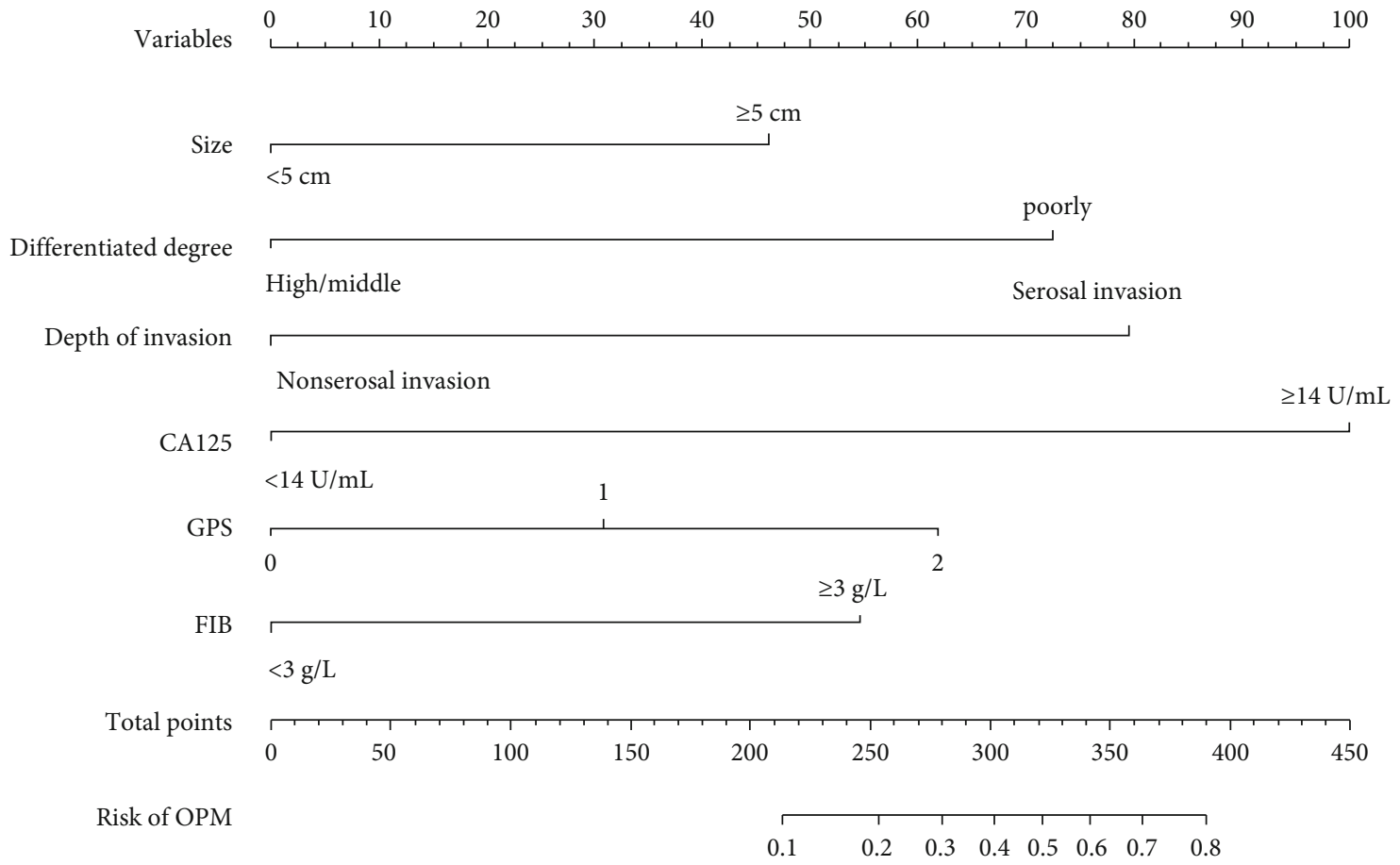

FIGURE 2: Nomogram for the probability of OPM based on the six predictors: tumor size, degree of differentiation, depth of invasion, GPS, and plasma levels of CA125 and fibrinogen. OPM: occult peritoneal metastasis; CA125: carbohydrate antigen 125; GPS: Glasgow prognosis score; FIB: fibrinogen.

TABLE 3: Coefficients of six variables in three nomogram models.

\begin{tabular}{|c|c|c|c|c|c|c|c|c|c|}
\hline \multirow{2}{*}{ Various } & \multicolumn{3}{|c|}{ Model A } & \multicolumn{3}{|c|}{ Model B } & \multicolumn{3}{|c|}{ Model C } \\
\hline & $\beta$ & OR (95\% CI) & $P$ & $\beta$ & OR (95\% CI) & $P$ & $\beta$ & OR (95\% CI) & $P$ \\
\hline Size $(\geq 5 \mathrm{~cm})$ & 0.839 & $2.313(1.130-4.737)$ & 0.022 & 1.194 & $3.300(1.802-6.043)$ & $<0.001$ & - & - & - \\
\hline $\begin{array}{l}\text { Differentiated degree } \\
\text { (poorly) }\end{array}$ & 1.420 & 4.137 (1.743-9.817) & 0.001 & 1.436 & $4.202(1.963-8.997)$ & $<0.001$ & - & - & - \\
\hline Serosal invasion & 1.669 & $\begin{array}{c}5.304(2.509- \\
11.214)\end{array}$ & $<0.001$ & 1.781 & $\begin{array}{c}5.938(3.028- \\
11.645)\end{array}$ & $<0.001$ & - & - & - \\
\hline CA125 ( $\geq 14 \mathrm{U} / \mathrm{mL})$ & 1.950 & $\begin{array}{c}7.026(3.541- \\
13.937)\end{array}$ & $<0.001$ & - & - & - & 2.171 & $\begin{array}{c}8.767(4.627- \\
16.613)\end{array}$ & $<0.001$ \\
\hline CA199 ( $\geq 30 \mathrm{U} / \mathrm{mL})$ & - & - & - & - & - & - & 0.741 & $2.098(1.127-3.904)$ & 0.019 \\
\hline GPS (2) & 1.339 & $\begin{array}{c}3.814(1.372- \\
10.599)\end{array}$ & 0.010 & - & - & - & 1.517 & $\begin{array}{c}4.558(1.829- \\
11.355)\end{array}$ & 0.001 \\
\hline FIB $(\geq 3 \mathrm{~g} / \mathrm{L})$ & 1.012 & $2.752(1.423-5.322)$ & 0.003 & - & - & - & 1.062 & $2.893(1.567-5.343)$ & 0.001 \\
\hline AIC & & 277.32 & & & 331.76 & & & 335.41 & \\
\hline
\end{tabular}

Model A: includes all six predictors; Model B: includes clinicopathological features only; Model C: includes hematologic characteristics only; CA199: carbohydrate antigen 19-9; CA125: carbohydrate antigen 125; GPS: Glasgow prognosis score; FIB: fibrinogen. AIC: Akaike information criterion.

\section{Discussion}

The average survival time of GC patients with peritoneal dissemination is less than six months, and these patients cannot achieve benefit from conventional therapy [24]. Typical signs, such as massive ascites or abdominal masses, are commonly used to diagnose peritoneal metastasis in patients with GC during clinical practice. However, these patients are often in the terminal stage of the disease and have poor clinical prognoses [7]. If peritoneal metastasis was accurately diagnosed in an early stage, the survival time of these patients would be improved. Unfortunately, the lack of specific and sensitive radiological examination approaches makes the accurate preoperative diagnosis of peritoneal metastasis of GC difficult [25-27]. In the absence of a single effective evaluation, there is a need for the construction of a model combining low cost and easily available laboratory parameters with radiological characteristics. The nomogram constructed in this study might provide a simple tool to predict the probability of OPM. This could be very useful for the clinical screening of high-risk populations for OPM and deciding whether or not to perform diagnostic laparoscopy. 


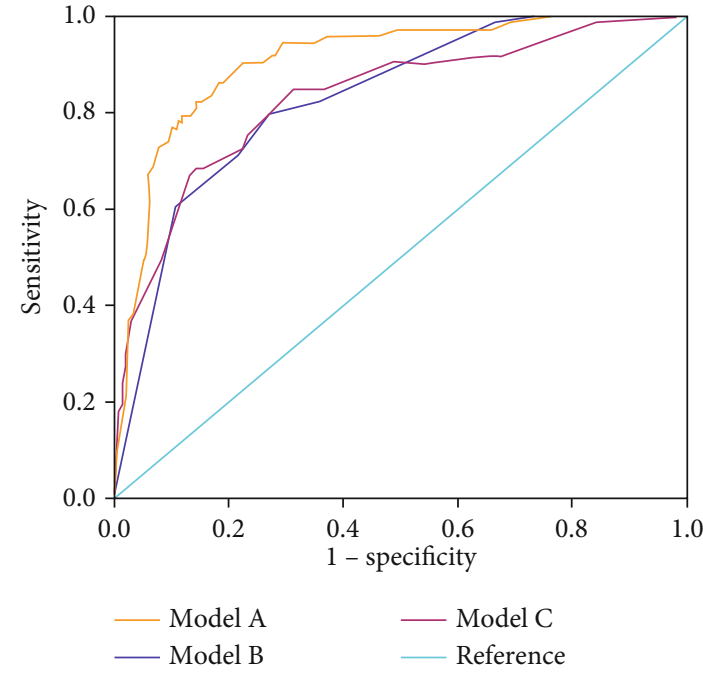

(a)

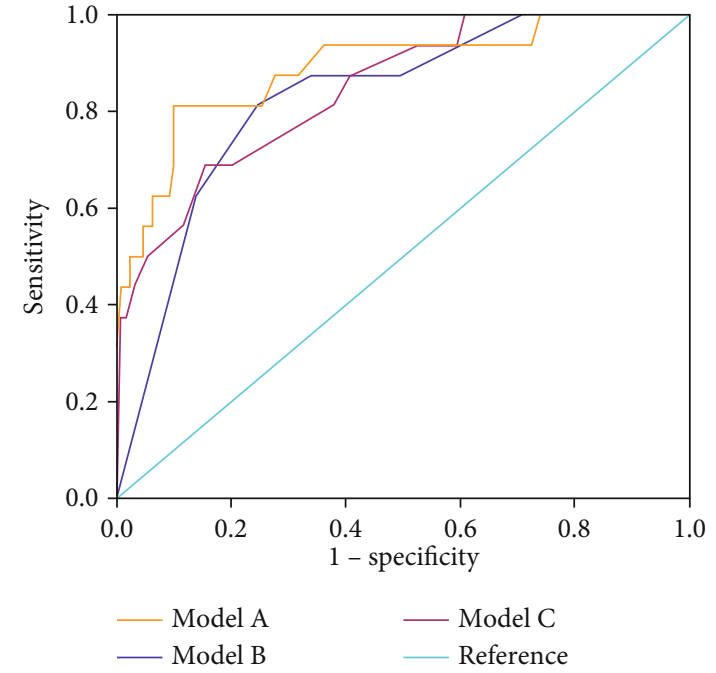

(b)

Figure 3: The discriminative abilities of Models A, B, and C were evaluated using the AUC of ROCs. Model A (including clinicopathological and preoperative hematological parameters) had the best performance both in the training (a) and validation (b) cohorts (DeLong's test, $P$ $<0.05)$.

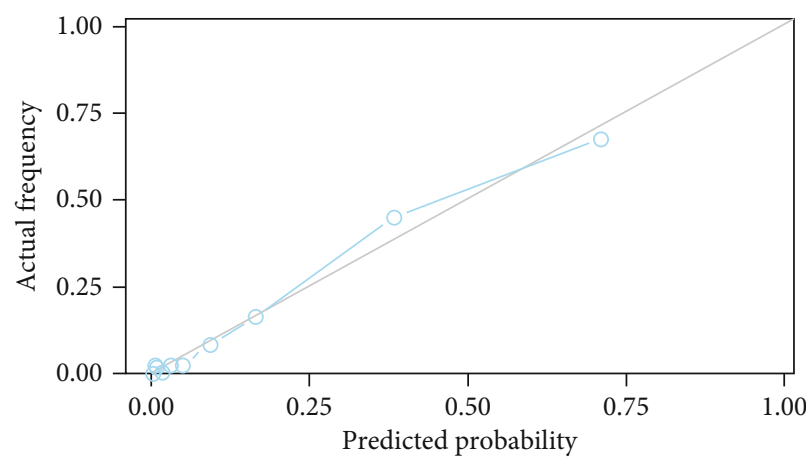

(a)

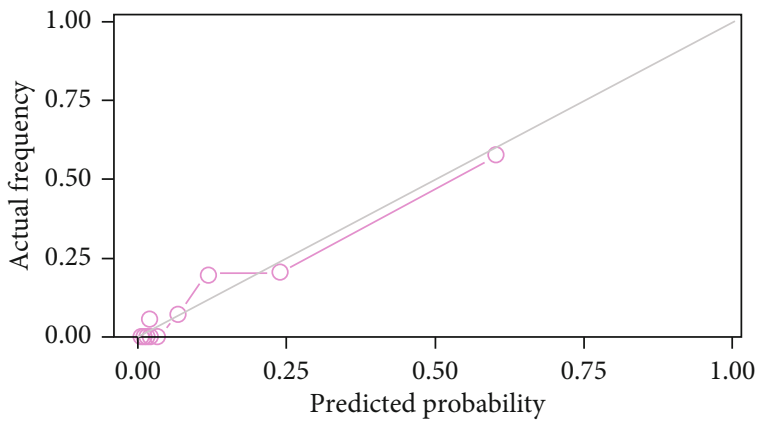

(b)

Figure 4: Nomogram calibration plots. (a) Calibration plot of the nomogram in the training set showed that the predicted and actual probabilities were similar. (b) In the validation cohort, the nomogram calibration plot revealed the consistency of the model (both Hosmer-Lemeshow's test, $P>0.05)$.

As a simple class of blood cell parameters, NLR and PLR could be easily determined from routine laboratory work and used to accurately reflect the level of systemic inflammation [28]. Preoperative NLR or PLR are strongly independent predictors of extensive peritoneal metastasis in advanced gastric cancer [29-31]. Although NLR and PLR were significantly correlated with OPM in this study, they were not included in the final nomogram as independent predictors of OPM. One reasonable explanation for this is that OPM might be considered the initial stage of extensive peritoneal metastasis lesions.

GPS is also an easily accessible and reliable marker that indirectly reflects the host inflammatory level using serum CRP and albumin. Patients with peritoneal metastases generally have higher CRP levels [32]. Such patients usually also have cachexia because of inadequate nutritional intake, frequent bleeding, or massive ascites, which aggravate hypoproteinemia. All of these may cause the GPS value to rise. Moreover, GPS was also closely correlated with the degree of peritoneal metastasis $(P=0.001)$ and volume of ascites $(P<0.001)$ [33]. This study also confirmed that GPS plays a key role in predicting OPM $(\mathrm{OR}=3.814)$.

The tiny isolated lesions in the peritoneal cavity mean that tumor biomarkers might be better tools than examination using images [34]. Preoperative plasma CA125 is one of the most reliable clinical markers in the diagnosis and prognosis evaluation of patients with peritoneal dissemination [35]. Emoto et al. reported that the sensitivity of CA125 for peritoneal metastasis at initial diagnosis was $45 \%$, and the median survival time of patients with lower CA125 levels was significantly longer than that of patients with higher levels [34]. In this study, higher serum CA125 was significantly correlated with OPM in patients with GC, and the odds ratio was significant at 5.355. Immunohistochemical analysis showed that CA125 expression on the surface of gastrointestinal malignant tumor cells was not common [36]. CA125, a large-molecule type-I transmembrane glycoprotein, only exists on the surface of some 


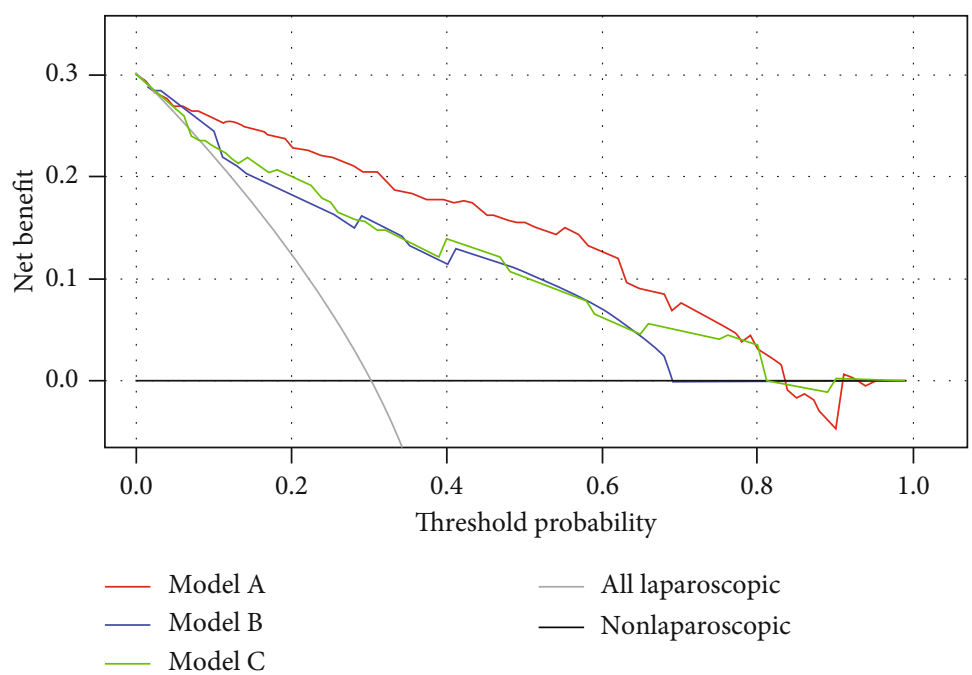

FIGURE 5: The net benefit curves for the three nomogram models in the training group. Model A had a positive net benefit when thresholds were between 0 and $82 \%$.

mesothelial cells which are the main cellular components of the peritoneum. This means that increased plasma CA125 level is not solely from the growth of the tumor focus, but reflective of the degree of peritoneal mesothelial cellular injury triggered by carcinomas [37].

The "seed and soil" hypothesis put forward by Paget [38] has been widely accepted as the fundamental theory of peritoneal dissemination in GC. In this theory, peritoneal-free cancer cells, which are detached from the primary tumor, are compared to "seeds" and the suitable environment of the tumor cancers are compared to "soil." The frequency of peritoneal metastasis should increase significantly because more free cancer cells are exfoliated from the primary tumor when the tumor cells penetrate the gastric serosa. Li et al. performed a prospective study of diagnostic laparoscopy for $249 \mathrm{cM} 0$ and suggested that the depth of invasion was an independent risk factor for intraperitoneal metastasis [39]. This study showed that $83.8 \%$ of OPM were cT4, indicating that tumor cells directly enter the abdominal cavity and adhere to the peritoneum to develop extensive peritoneal metastasis. Interestingly, Yoshida and Huang et al. [40, 41] reported a small number of early non-serosa-invasive cases with peritoneal dissemination and provided a novel possible mechanism through which primary tumor cells could be indirectly shed into the peritoneal cavity via lymphatic networks on the peritoneum.

As a rising technology in recent years, radiomics has the advantages of being noninvasive and producing large volumes of information. Recently, Dong et al. developed an OPM prediction model based on radiomics features, including three predictors (RS1, RS2, and Lauren's type), and the model showed an excellent ability to predict OPM $(A U C=0.958)$ [7]. With the continuous improvement of detection equipment and algorithm technology, a promising diagnostic method for OPM could arise from combining radiologic characteristics and other clinicopathological parameters $[42,43]$.

\section{Conclusion}

Both clinicopathological and preoperative hematological parameters are significantly associated with OPM. The nomogram constructed in this study could effectively predict the incidence of OPM. It is helpful to identity high-risk patients with OPM and provide a guide for optimal treatment strategies and avoid unnecessary operative treatments. However, there are some limitations in the study. Due to the small size of the samples in the validation set, the baseline characteristics were not well-matched between the training and validation sets. Another limitation to this study was that it was a single-center study with retrospective data collection. Therefore, the influence of selection bias should be considered when using this prediction nomogram in other centers.

\section{Data Availability}

The data used to support the findings of this study are available from the corresponding author upon reasonable request.

\section{Conflicts of Interest}

The authors declare that they have no competing interests.

\section{Authors' Contributions}

Yongbin Zheng and Chao Yang contributed in the conception and design of the study. Chao Yang, Yujie Yang, and Xiaodong Huang contributed in the collection and assembly of data. Chao Yang, Huali Li, Huangrong Cheng, and Shilun Tong contributed in data analysis and interpretation. Chao Yang and Yongbin Zheng contributed in manuscript writing. All authors gave final approval of the manuscript. 


\section{References}

[1] F. Bray, J. Ferlay, I. Soerjomataram, R. L. Siegel, L. A. Torre, and A. Jemal, "Global cancer statistics 2018: GLOBOCAN estimates of incidence and mortality worldwide for 36 cancers in 185 countries," CA: A Cancer Journal for Clinicians, vol. 68, no. 6, pp. 394-424, 2018.

[2] I. Nakayama, K. Chin, T. Matsushima et al., "Retrospective comparison of S-1 plus cisplatin versus S-1 monotherapy for the treatment of advanced gastric cancer patients with positive peritoneal cytology but without gross peritoneal metastasis," International Journal of Clinical Oncology, vol. 22, no. 6, pp. 1060-1068, 2017.

[3] S. J. Kim, H. H. Kim, Y. H. Kim et al., "Peritoneal metastasis: detection with 16- or 64-detector row CT in patients undergoing surgery for gastric cancer," Radiology, vol. 253, no. 2, pp. 407-415, 2009.

[4] S. Burbidge, K. Mahady, and K. Naik, "The role of CT and staging laparoscopy in the staging of gastric cancer," Clinical Radiology, vol. 68, no. 3, pp. 251-255, 2013.

[5] X. Wu, Z. Li, Z. Li et al., "Hyperthermic intraperitoneal chemotherapy plus simultaneous versus staged cytoreductive surgery for gastric cancer with occult peritoneal metastasis," Journal of Surgical Oncology, vol. 111, no. 7, pp. 840-847, 2015.

[6] T. Yamaguchi, A. Takashima, K. Nagashima et al., "Efficacy of postoperative chemotherapy after resection that leaves no macroscopically visible disease of gastric cancer with positive peritoneal lavage cytology (CY1) or localized peritoneum metastasis (P1a): a multicenter retrospective study," Annals of Surgical Oncology, vol. 27, no. 1, pp. 284-292, 2020.

[7] D. Dong, L. Tang, Z. Y. Li et al., "Development and validation of an individualized nomogram to identify occult peritoneal metastasis in patients with advanced gastric cancer," Annals of Oncology, vol. 30, no. 3, pp. 431-438, 2019.

[8] N. Yamada, A. Akai, Y. Nomura, and N. Tanaka, "The impact and optimal indication of non-curative gastric resection for stage IV advanced gastric cancer diagnosed during surgery: 10 years of experience at a single institute," World Journal of Surgical Oncology, vol. 14, no. 1, p. 79, 2016.

[9] R. M. Kwee and T. C. Kwee, "Modern imaging techniques for preoperative detection of distant metastases in gastric cancer," World Journal of Gastroenterology, vol. 21, no. 37, pp. 1050210509, 2015.

[10] M. Fairweather, K. Jajoo, N. Sainani, M. M. Bertagnolli, and J. Wang, "Accuracy of EUS and CT imaging in preoperative gastric cancer staging," Journal of Surgical Oncology, vol. 111, no. 8, pp. 1016-1020, 2015.

[11] Y. Honma, T. Terauchi, U. Tateishi et al., "Imaging peritoneal metastasis of gastric cancer with $18 \mathrm{~F}$-fluorothymidine positron emission tomography/computed tomography: a proof-ofconcept study," The British Journal of Radiology, vol. 91, no. 1089, article 20180259, 2018.

[12] Z. Li, Z. Li, L. Zhang et al., "Staging laparoscopy for locally advanced gastric cancer in Chinese patients: a multicenter prospective registry study," BMC Cancer, vol. 18, no. 1, p. 63, 2018.

[13] N. Ikoma, M. Blum, Y. J. Chiang et al., "Yield of staging laparoscopy and lavage cytology for radiologically occult peritoneal carcinomatosis of gastric cancer," Annals of Surgical Oncology, vol. 23, no. 13, pp. 4332-4337, 2016.

[14] B. Badgwell, P. Das, and J. Ajani, "Treatment of localized gastric and gastroesophageal adenocarcinoma: the role of accurate staging and preoperative therapy," Journal of Hematology \& Oncology, vol. 10, no. 1, p. 149, 2017.

[15] L. X. Zhang, Z. J. Wei, A. M. Xu, and J. H. Zang, "Can the neutrophil-lymphocyte ratio and platelet-lymphocyte ratio be beneficial in predicting lymph node metastasis and promising prognostic markers of gastric cancer patients? Tumor maker retrospective study," International Journal of Surgery, vol. 56, pp. 320-327, 2018.

[16] K. Wang, F. Diao, Z. Ye et al., "Prognostic value of systemic immune-inflammation index in patients with gastric cancer," Chinese Journal of Cancer, vol. 36, no. 1, p. 75, 2017.

[17] H. Xie, G. Yuan, S. Huang et al., "The prognostic value of combined tumor markers and systemic immune-inflammation index in colorectal cancer patients," Langenbeck's Archives of Surgery, vol. 405, no. 1, pp. 1119-1130, 2020.

[18] S. Yang, K. Zhao, X. Ding, H. Jiang, and H. Lu, "Prognostic significance of hematological markers for patients with nasopharyngeal carcinoma: a meta-analysis," Journal of Cancer, vol. 10, no. 11, pp. 2568-2577, 2019.

[19] C. Wang, W. He, Y. Yuan et al., "Comparison of the prognostic value of inflammation-based scores in early recurrent hepatocellular carcinoma after hepatectomy," Liver International, vol. 40, no. 1, pp. 229-239, 2019.

[20] Y. Okugawa, Y. Toiyama, A. Yamamoto et al., "Lymphocyteto-C-reactive protein ratio and score are clinically feasible nutrition-inflammation markers of outcome in patients with gastric cancer," Clinical Nutrition, vol. 39, no. 4, pp. 12091217, 2020.

[21] R. Yang, Q. Chang, X. Meng, N. Gao, and W. Wang, "Prognostic value of systemic immune-inflammation index in cancer: a metaanalysis," Journal of Cancer, vol. 9, no. 18, pp. 3295-3302, 2018.

[22] O. Repetto and V. De Re, "Coagulation and fibrinolysis in gastric cancer," Annals of the New York Academy of Sciences, vol. 1404, no. 1, pp. 27-48, 2017.

[23] H. Shimada, T. Noie, M. Ohashi, K. Oba, and Y. Takahashi, "Clinical significance of serum tumor markers for gastric cancer: a systematic review of literature by the Task Force of the Japanese Gastric Cancer Association," Gastric Cancer, vol. 17, no. 1, pp. 26-33, 2014.

[24] B. W. Loggie and P. Thomas, "Gastrointestinal cancers with peritoneal carcinomatosis: surgery and hyperthermic intraperitoneal chemotherapy," Oncology (Williston Park), vol. 29, no. 7, pp. 515-521, 2015.

[25] P. Perlaza, J. Ortín, M. Pagès et al., "Should 18F-FDG PET/CT be routinely performed in the clinical staging of locally advanced gastric adenocarcinoma?," Clinical Nuclear Medicine, vol. 43, no. 6, pp. 402-410, 2018.

[26] J. W. Kim, S. S. Shin, S. H. Heo et al., "The role of threedimensional multidetector CT gastrography in the preoperative imaging of stomach cancer: emphasis on detection and localization of the tumor," Korean Journal of Radiology, vol. 16, no. 1, pp. 80-89, 2015.

[27] B. Menassel, A. Duclos, G. Passot et al., "Preoperative CT and MRI prediction of non-resectability in patients treated for pseudomyxoma peritonei from mucinous appendiceal neoplasms," European Journal of Surgical Oncology, vol. 42, no. 4, pp. 558-566, 2016.

[28] Y. Jiang, H. Xu, H. Jiang, S. Ding, and T. Zheng, "Pretreatment neutrophil-lymphocyte count ratio may associate with gastric cancer presence," Cancer Biomarkers, vol. 16, no. 4, pp. 523$528,2016$. 
[29] Y. Wu, M. Jiang, Y. Qin, F. Lin, and M. Lai, "Single and combined use of neutrophil-lymphocyte ratio, platelet-lymphocyte ratio and carcinoembryonic antigen in diagnosing gastric cancer," Clinica Chimica Acta, vol. 481, pp. 20-24, 2018.

[30] H. Saito, Y. Kono, Y. Murakami et al., "Prognostic significance of platelet-based inflammatory indicators in patients with gastric cancer," World Journal of Surgery, vol. 42, no. 8, pp. 25422550, 2018.

[31] N. Nakamura, S. Kinami, Y. Fujii et al., "The neutrophil/lymphocyte ratio as a predictor of peritoneal metastasis during staging laparoscopy for advanced gastric cancer: a retrospective cohort analysis," World Journal of Surgical Oncology, vol. 17, no. 1, p. 108, 2019.

[32] Y. Nakayama, N. Gotohda, H. Shibasaki, S. Nomura, T. Kinoshita, and R. Hayashi, "Usefulness of the neutrophil/lymphocyte ratio measured preoperatively as a predictor of peritoneal metastasis in patients with advanced gastric cancer," Surgery Today, vol. 44, no. 11, pp. 2146-2152, 2014.

[33] S. Q. Yuan, R. C. Nie, Y. M. Chen et al., "Glasgow Prognostic Score is superior to ECOG PS as a prognostic factor in patients with gastric cancer with peritoneal seeding," Oncology Letters, vol. 15, no. 4, pp. 4193-4200, 2018.

[34] S. Emoto, H. Ishigami, H. Yamashita, H. Yamaguchi, S. Kaisaki, and J. Kitayama, "Clinical significance of CA125 and CA72-4 in gastric cancer with peritoneal dissemination," Gastric Cancer, vol. 15, no. 2, pp. 154-161, 2012.

[35] D. H. Kim, H. Y. Yun, D. H. Ryu et al., "Preoperative CA 125 is significant indicator of curative resection in gastric cancer patients," World Journal of Gastroenterology, vol. 21, no. 4, pp. 1216-1221, 2015.

[36] J. L. Dennis, T. R. Hvidsten, E. C. Wit et al., "Markers of adenocarcinoma characteristic of the site of origin: development of a diagnostic algorithm," Clinical Cancer Research, vol. 11, no. 10, pp. 3766-3772, 2005.

[37] S. Bafna, S. Kaur, and S. K. Batra, "Membrane-bound mucins: the mechanistic basis for alterations in the growth and survival of cancer cells," Oncogene, vol. 29, no. 20, pp. 2893-2904, 2010.

[38] M. Akhtar, A. Haider, S. Rashid, and A. D. M. H. Al-Nabet, "Paget's "Seed and Soil" Theory of Cancer Metastasis: An Idea Whose Time has Come," Advances In Anatomic Pathology, vol. 26, no. 1, pp. 69-74, 2019.

[39] Z. Li, Z. Li, S. Jia et al., "Depth of tumor invasion and tumoroccupied portions of stomach are predictive factors of intraabdominal metastasis," Chinese Journal of Cancer Research, vol. 29, no. 2, pp. 109-117, 2017.

[40] M. Yoshida, T. Sugino, K. Kusafuka et al., "Peritoneal dissemination in early gastric cancer: importance of the lymphatic route," Virchows Archiv, vol. 469, no. 2, pp. 155-161, 2016.

[41] B. Huang, Z. Sun, Z. Wang et al., "Factors associated with peritoneal metastasis in non-serosa-invasive gastric cancer: a retrospective study of a prospectively-collected database," BMC Cancer, vol. 13, no. 1, p. 57, 2013.

[42] W. Huang, K. Zhou, Y. Jiang et al., "Radiomics nomogram for prediction of peritoneal metastasis in patients with gastric cancer," Frontiers in Oncology, vol. 10, p. 1416, 2020.

[43] Z. Y. Li, L. Tang, Z. M. Li et al., "Four-point computed tomography scores for evaluation of occult peritoneal metastasis in patients with gastric cancer: a region-to-region comparison with staging laparoscopy," Annals of Surgical Oncology, vol. 27, no. 4, pp. 1103-1109, 2020. 